\title{
On the Network Topology of Crosslinked Acrylate Photopolymers: A Molecular Dynamics Case Study
}

John J. Karnes, * Todd H. Weisgraber, James S. Oakdale, Magi Mettry, Maxim Shusteff, and Juergen Biener

Lawrence Livermore National Laboratory, 7000 East Avenue, Livermore, CA, USA

\section{Supporting Information}

\section{S1. Simulated Polymerization and Computational Details}

As noted in the main text, all-atom molecular dynamics simulations were performed in the study

of three representative acrylate monomers. All simulations were run on LLNL's Quartz

supercomputer, which consists of 3018 total Intel Xenon E5-2695 nodes, with 36 cores and 128

GB of CPU memory per node. Table S1 contains additional details regarding simulation sizes and performance.

Table S1. Reactive MD simulation sizes, applied computational resources, and performance.

\begin{tabular}{|c|c|c|c|c|}
\hline & atoms/molecule & atoms/simulation & nodes & ns/day \\
\hline PETA & 39 & 78000 & 20 & 10 \\
\hline HDDA & 34 & 68000 & 18 & 14 \\
\hline PEGDA & 36 & 72000 & 18 & 13 \\
\hline
\end{tabular}


We include representative spatial mass density maps of the three systems to show that the liquid monomer simulation boxes are equilibrated and well-formed. The density maps in Figure S1 represent average densities collected with $5 \AA \times 5 \AA$ grid spacing of the (arbitrarily selected) $z$ plane, averaged over a $1 \mathrm{~ns}$ trajectory. Differences in the mapping roughness are mostly function of the respective monomers' self-diffusion rates (see main text for computed values).

(a)

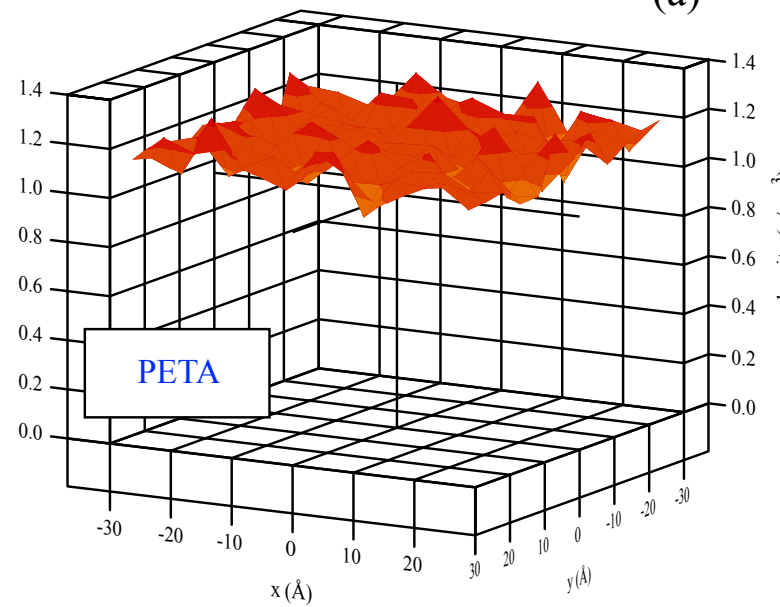

(c)

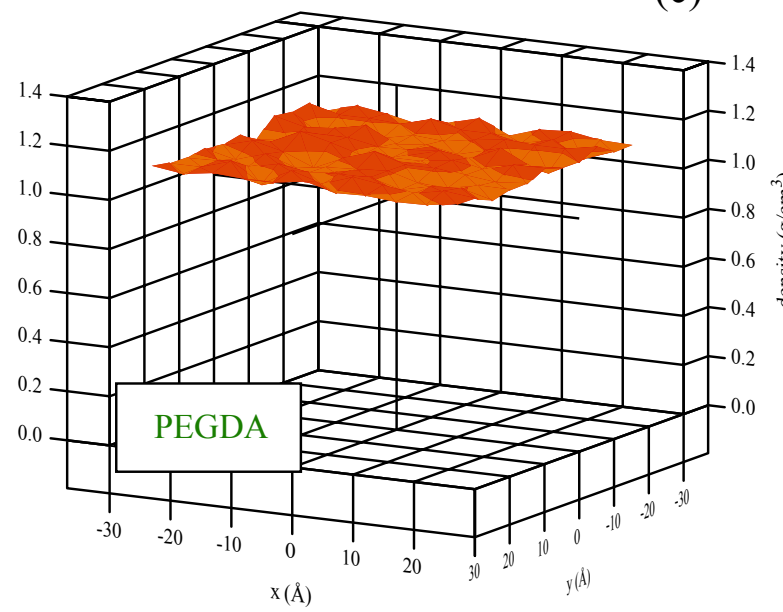

Figure S1. Spatial density maps of (a) PETA, (b) HDDA, and (c) PEGDA neat liquid monomer simulation boxes. (b)
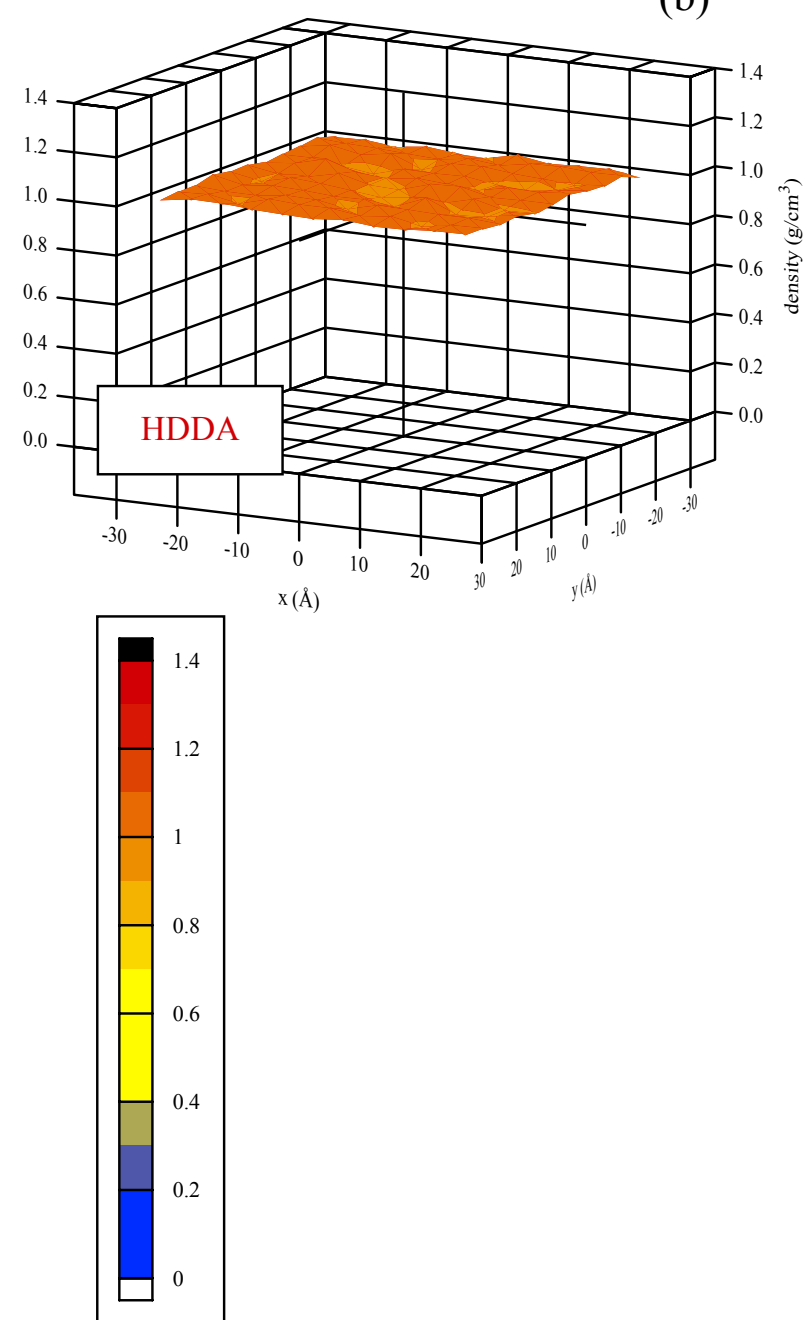
In these simulations the REACTER $^{1}$ protocol scans the simulation box for three potential reactions:

1) Propagation: acrylate radical (monomer) + acrylate

2) Propagation: acrylate radical (oligomer+) + acrylate

3) Termination: acrylate radical + acrylate radical

Reactions 1 and 2 are distinct since the local affected force field topology of the radical-bearing acrylate is slightly different depending on whether or not the acrylate site with the radical has previously participated in a reaction.

(a)

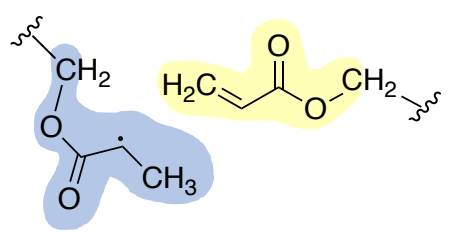

(b)

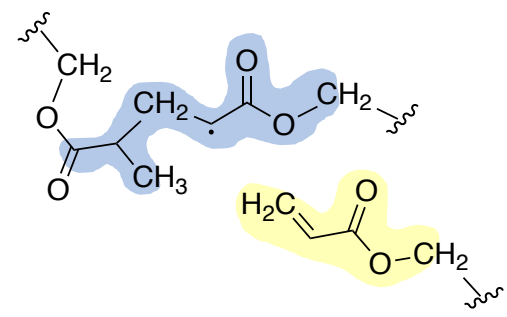

Figure S2. Acrylate-acrylate radical polymerization requires two distinct templates depending on whether the radical-bearing acrylate group is (a) part of a free, unreacted acrylate moiety or (b) part of a propagating chain. Blue highlighting indicates all atoms within 3 bonds of the radical carbon and yellow highlighting indicates all atoms within 3 bonds of the acrylate alkene carbons. 
Figure S3 shows the double bond conversion versus simulation time for all three polymer systems. The Quartz supercomputer limits per-job wall time to 24 hours. Trajectories were collected for each starting configuration were collected for 3 days of wall time (see Table S1 for performance/monomer/day.) After the conversion rates began to plateau, higher conversions were achieved by extending the cutoff radius $r$ from 4.0 to $4.5 \AA$. This adjustment served to free some trapped or sterically inaccessible acrylate monomers and allow for higher conversion in the regime beyond the gel point conversions, a perturbation analogous to the post curing or post irradiation of the photopolymerized additively manufactured materials that inspire these simulations. This change in cutoff resulted in no detectible change in the polymer network structures, which would emerge as discontinuities in the network properties explored in this work.

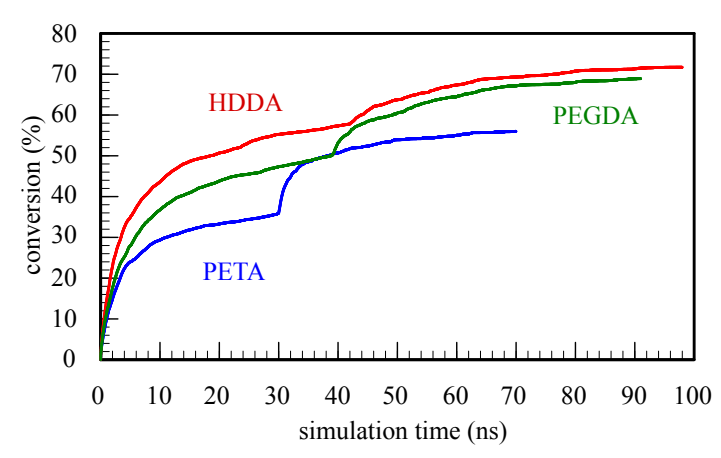

Figure S3. Double bond conversion versus simulation time.

As polymerization progresses, van der Waals interactions are replaced by covalent bonds and the global volume of the system decreases. Performing simulations at ambient temperature and in the NPT ensemble allows us to observe the system's volume change as a function of conversion on-the-fly. Figure S4 shows the shrinkage percentage versus conversion for the three systems. The initial linear relationship between volume reduction as a function of of double bond conversion functions serves as a self-consistent check against hysteresis effects since the systems polymerize 
at significantly different rates throughout the course of the simulation, both relative to themselves within the same simulations and when compared to dissimilar monomer systems.

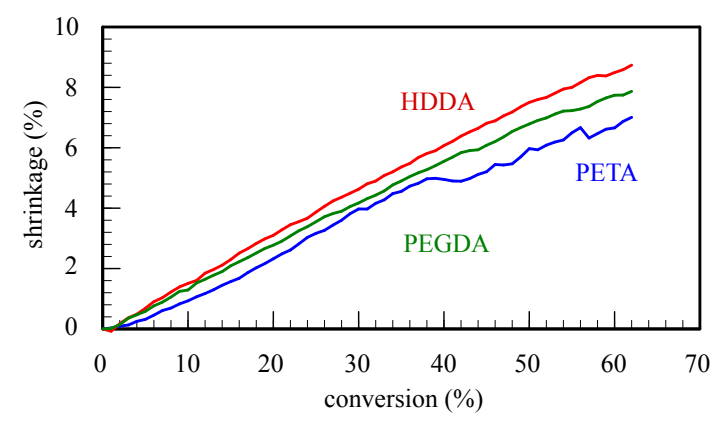

Figure S4. Volume reduction versus conversion.

\section{S2. Free and Reacted Acrylates}

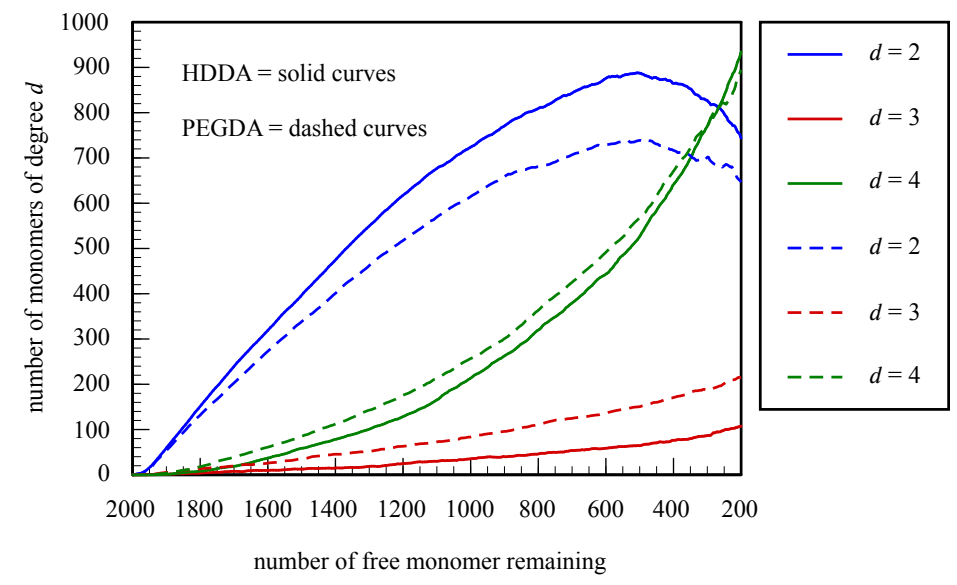

Figure S5. Evolving HDDA and PEGDA degree histograms as a function of remaining free monomers looks similar to simply overlaying the plots from Figure $7 \mathrm{~b}$ and $7 \mathrm{c}$. This is because the acrylate and monomer conversion curves for HDDA and PEGDA track within a few percent of each other, as shown in Figure 5. 
Acrylate-acrylate radial distribution functions summarize the local spatial ordering in the simulation cells. Data for the PEGDA system was presented in the main text and is presented here with expanded $x$-axes in Figure S6 and again in Figure S7 with corresponding plots for HDDA and PETA, mostly highlighting the similarity in local ordering. We expect that future coarsegrained simulations that access significantly larger length scales will reveal differences not present in these all-atom simulations. ${ }^{2}$
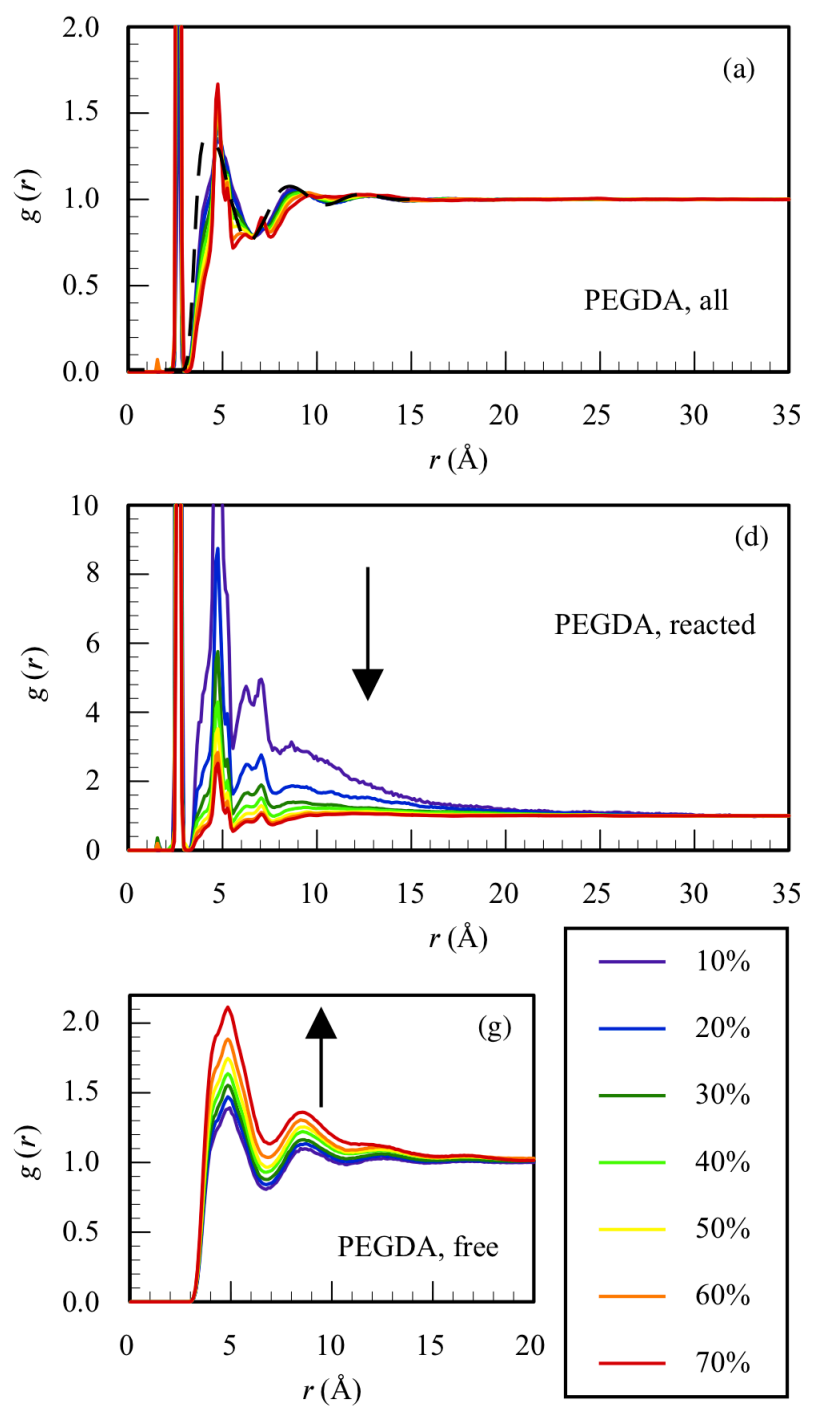

Figure S6. Acrylate-acrylate radial distribution functions at selected conversions for the PEGDA system. All acrylate groups in the system are accounted for in panel (a). Panel (b) shows $g(r)$ only 
for acrylate groups whose vinyl group has reacted and panel (c) shows the free, unreacted acrylate groups.
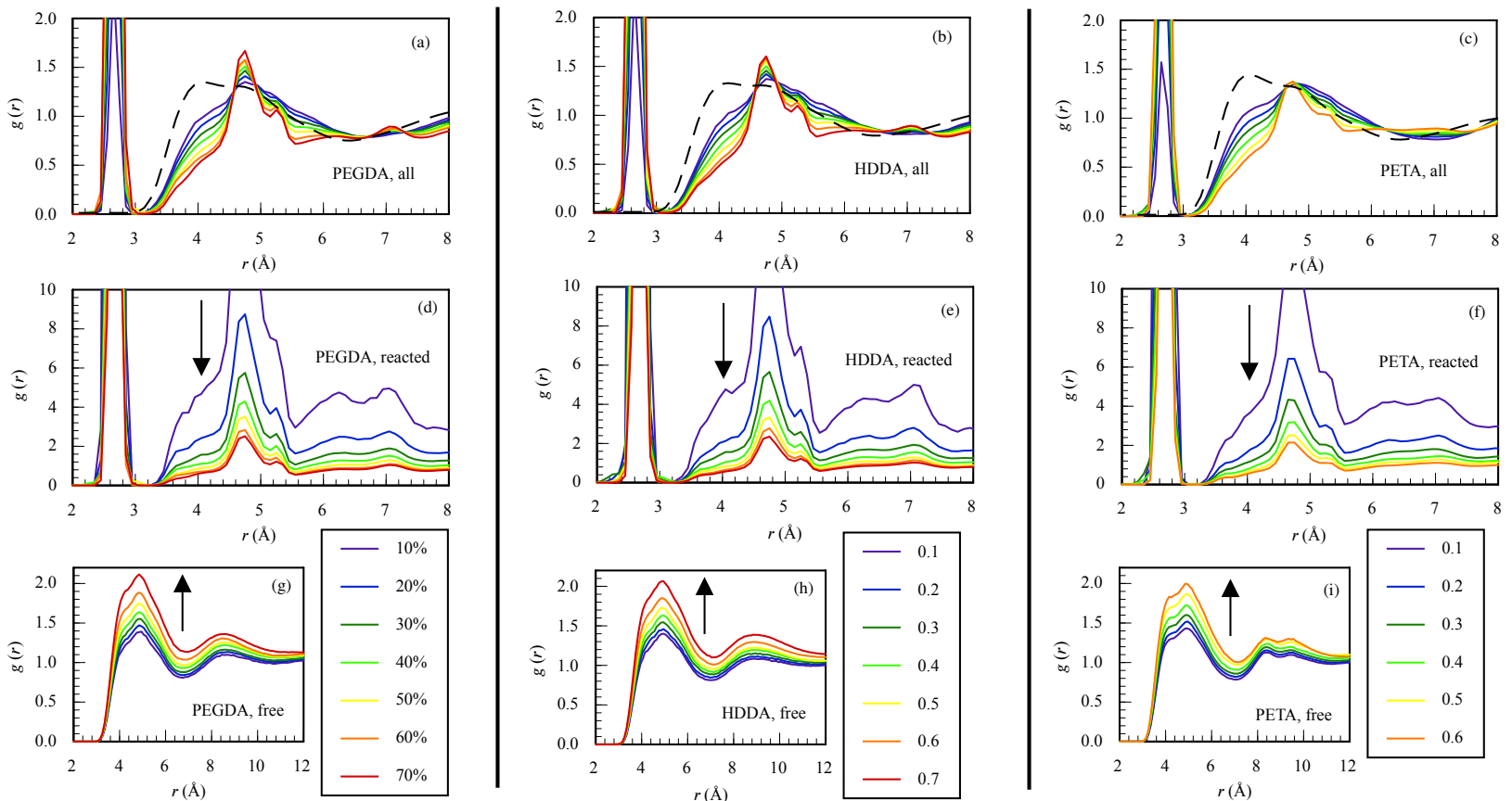

Figure S7. Acrylate-acrylate radial distribution functions at selected conversions. All acrylate groups in each respective system are accounted for in panels (a-c). Panels (d-f) show $g(r)$ only for acrylate groups whose vinyl group has reacted and panels (g-i) show the free, unreacted acrylate groups.

\section{REFERENCES}

(1) Gissinger, J. R.; Jensen, B. D.; Wise, K. E. Modeling Chemical Reactions in Classical Molecular Dynamics Simulations. Polymer 2017, 128, 211-217.

(2) Jankowski, E.; Ellyson, N.; Fothergill, J. W.; Henry, M. M.; Leibowitz, M. H.; Miller, E. D.; Alberts, M.; Chesser, S.; Guevara, J. D.; Jones, C. D.; et al. Perspective on Coarse-Graining, Cognitive Load, and Materials Simulation. Comput. Mater. Sci. 2020, 171, 109129. 